\title{
Flutter Instability Speeds of Guided Splined Disks: An Experimental and Analytical Investigation
}

\author{
Ahmad Mohammadpanah and Stanley G. Hutton \\ Department of Mechanical Engineering, The University of British Columbia, Vancouver, BC, Canada V6T 1Z4 \\ Correspondence should be addressed to Ahmad Mohammadpanah; ahmadpa20@gmail.com
}

Received 30 October 2014; Accepted 11 February 2015

Academic Editor: Mickaël Lallart

Copyright ( 2015 A. Mohammadpanah and S. G. Hutton. This is an open access article distributed under the Creative Commons Attribution License, which permits unrestricted use, distribution, and reproduction in any medium, provided the original work is properly cited.

\begin{abstract}
"Guided splined disks" are defined as flat thin disks in which the inner radius of the disk is splined and matches a splined arbor that provides the driving torque for rotating the disk. Lateral constraint for the disk is provided by space fixed guide pads. Experimental lateral displacement of run-up tests of such a system is presented, and the flutter instability zones are identified. The results indicate that flutter instability occurs at speeds when a backward travelling wave of a mode meets a reflected wave of a different mode. Sometimes, the system cannot pass a flutter zone, and transverse vibrations of the disk lock into that flutter instability zone. The governing linear equations of transverse motion of such a spinning disk, with assumed free inner and outer boundary conditions, are derived. A lateral constraint is introduced and modeled as a linear spring. Rigid body translational and tilting degrees of freedom are included in the analysis of the total motion of the spinning disk. The eigenvalues of the system are computed numerically, and the flutter instability zones are defined. The results show that the mathematical model can predict accurately the flutter instability zones measured in the experimental tests.
\end{abstract}

\section{Introduction}

Splined disks find extensive application in the forest products industry as circular saws. In recent years guided spline arbor saws have been universally used in North America. This is due to their superior performance compared with clamped saws [1]. In a clamped saw the blade is rigidly fixed to the driving arbor, while in a splined saw the inner radius of the blade is splined and matches a splined arbor that provides the driving torque for the saw. The splined saw thus has the ability to move laterally and tilt on the driving arbor. Lateral constraint for these saws is provided by space fixed guide pads which constrain the lateral motion of the saw. In practice the maximum cutting speed for a clamped saw coincides with the lowest critical speed of the rotating disk, whereas the splined saw is able to operate at speeds in excess of the lowest critical speed [1].

A large portion of the existing literature on this subject is concerned with the vibration characteristics of clamped disks. The number of studies relating to guided spline disks is limited.
$\mathrm{Mu}$ and Chen [2] studied the effects of the location, number, and stiffness of guide pads on the lowest critical rotational frequencies of the rotating circular clamped saws. They considered the influence of the cutting temperature in their analysis. They concluded that the introduction of one guide does not stably increase critical rotational speeds of the circular saw.

Khorasany and Hutton [3] conducted experiments to investigate the effect of large deformations on the frequency characteristics of a clamped disk. The disk in their investigations was subjected to a space fixed external force provided by an air jet. They found that, depending on the level of nonlinearity, some of the frequency paths around the critical speed level off and maintain a nearly constant value. They also found that, at the speed at which the stationary wave collapses, a sudden change in the measured frequencies is notable.

Khorasany and Hutton [4] numerically investigated the effect of geometrical nonlinearity on the frequency characteristics of a spinning disk. They discretized the governing equations of motion, found the equilibrium solutions, and then 
linearized the governing equations around these solutions. They verified that there is a close correspondence between the numerically and experimentally obtained results.

Vogel and Skinner [5] developed the frequency determinants for various combinations of boundary conditions associated with the transverse vibrations of a disk. They derived the values of the resonant frequencies for different modes.

Chen et al. [6] investigated the possibility of secondary resonance of a spinning disk under space fixed excitations. They employed the multiple scale method to predict the steady state solutions. They investigated the nonlinear coupling between forward and backward travelling waves. They found that combination resonance may occur when two travelling waves couple, and when the combination resonance occurs, the frequencies of the free oscillation components are shifted slightly from the respective natural frequencies of the forward and backward wave.

Price [7] studied analytically the dynamic characteristics of a spinning disk that is free to translate while its central clamping collar remains perpendicular to the axis of rotation. He studied the effect of a rigid body translational degree of freedom on the dynamic response of rotating disks.

Chen and Bogy [8] investigated the effect of rigid body tilting on the stability of rotating disks subjected to a rotating load system. They studied the effects of different load parameters such as friction force, transverse mass, damping, and stiffness of a stationary load system in contact with the spinning disk on the stability of the system. They concluded that the interaction between the natural frequencies of backward and reflected travelling waves causes flutter type instability for an elastically constrained disk.

Chen and Wong [9] studied the vibration and stability of a spinning disk with axial spindle displacement in contact with a number of fixed springs. They used a finite element method to calculate the eigenvalues of the coupled system. In their study they investigate the behavior of modal interactions when two modes are almost degenerate. They concluded that when the frequencies of the neighboring modes are away from zero, the behavior of modal interaction is similar to those of a conventional disk without axial translation.

Khorasany and Hutton [10] investigated analytically the stability characteristics of guided spinning disks having rigid body translational degrees of freedom and included nonlinear geometric effects. They found that, because of the effect of the rigid body translational degree of freedom, divergence instability does not occur. They concluded that in this case the guided disk still has the same critical speeds as the unguided disk. They proved analytically that the interaction of a forward or backward traveling wave with the rigid body translational mode does not induce flutter type instability for a disk with rigid body translational degree of freedom.

Yang [11] investigated the effect of rigid body degrees of freedom on the stability of spline guided disks. He concluded that stable operation of the splined disk beyond critical speed is possible. There is limited available literature on prediction of flutter instability of a splined guided disk.
Recently Khorasany et al. [12] investigated the idling dynamic characteristics of guided spline circular saws experimentally and numerically. They conducted experimental tests on the frequency characteristics of a guided splined saw subjected to large lateral deflection. They found that the blade frequencies show a significant change as a result of the initial lateral displacement imposed by the external force. It was also found that, due to the presence of the external force, a stationary wave develops and collapses at a higher speed. For the numerical simulations, they used the nonlinear governing equations based on von Kármán plate theory. They investigated the effect of nonlinearity on the amplitude and frequency response of the guided blade.

In this paper, experimental tests are first conducted for a guided splined disk. Transverse response of the disk during run-up is analyzed in time and frequency domains. Then, eigenvalues of the system are computed and the flutter regions are identified. Numerical and experimental predictions of flutter zones are compared and discussed. The term "lockedin flutter instability" is defined to be a case where the disk cannot pass a flutter zone. The maximum stable operation speed of a guided splined disk is defined as the start of a locked-in flutter. The effect of geometrical properties of the disk on the start of locked-in flutter instability is analyzed.

\section{Experimental Tests and Results}

Experimental test facilities were developed which enable the measurement of natural frequencies of a guided splined disk as a function of rotation speed. The experimental setup is presented in Figure 1. To measure the disk deflection, a noncontacting inductance probe was used. Electromagnetic excitation was used to provide white noise excitation over the frequency range $1-100 \mathrm{~Hz}$. To investigate the dynamic characteristics of a rotating disk when subjected to the effect of a stationary lateral constant force, an air jet was used. The magnitude of the air jet force was kept at a constant value by keeping a constant air pressure. Results were obtained by measuring the vibration responses of the disk at the location of the displacement probe as the speed is ramped up from $0 \mathrm{rpm}$ to $4,000 \mathrm{rpm}$ at a constant rate over $600 \mathrm{~s}$.

Figure 2 shows the displacement results of a disk with inner and outer diameter of $200 \mathrm{~mm}$ and $700 \mathrm{~mm}$, respectively. The thickness of the disk under investigations was $2.8 \mathrm{~mm}$. The disk was made out of steel. Figure 2 shows the deflection of the disk measured by the displacement probe during run-up from 0 to $3600 \mathrm{rpm}$ at constant acceleration (note that the test had to be stopped at $3600 \mathrm{rpm}$ due to very large transverse vibrations of the disk). The variation of the excited frequencies of the disk, as a function of rotation speed, was computed and plotted in a form of frequencyspeed color map (Figure 3). Frequency color maps of the power spectrum illustrate the variation of disk frequencies with rotation speed. It illustrates the energy of the signal at each speed and frequency with a color spectrum.

The experimental results show that the blade suffers two minor critical speeds at about $1500 \mathrm{rpm}$ and $2100 \mathrm{rpm}$. It experiences locked-in flutter vibrations at about $2400 \mathrm{rpm}$. 

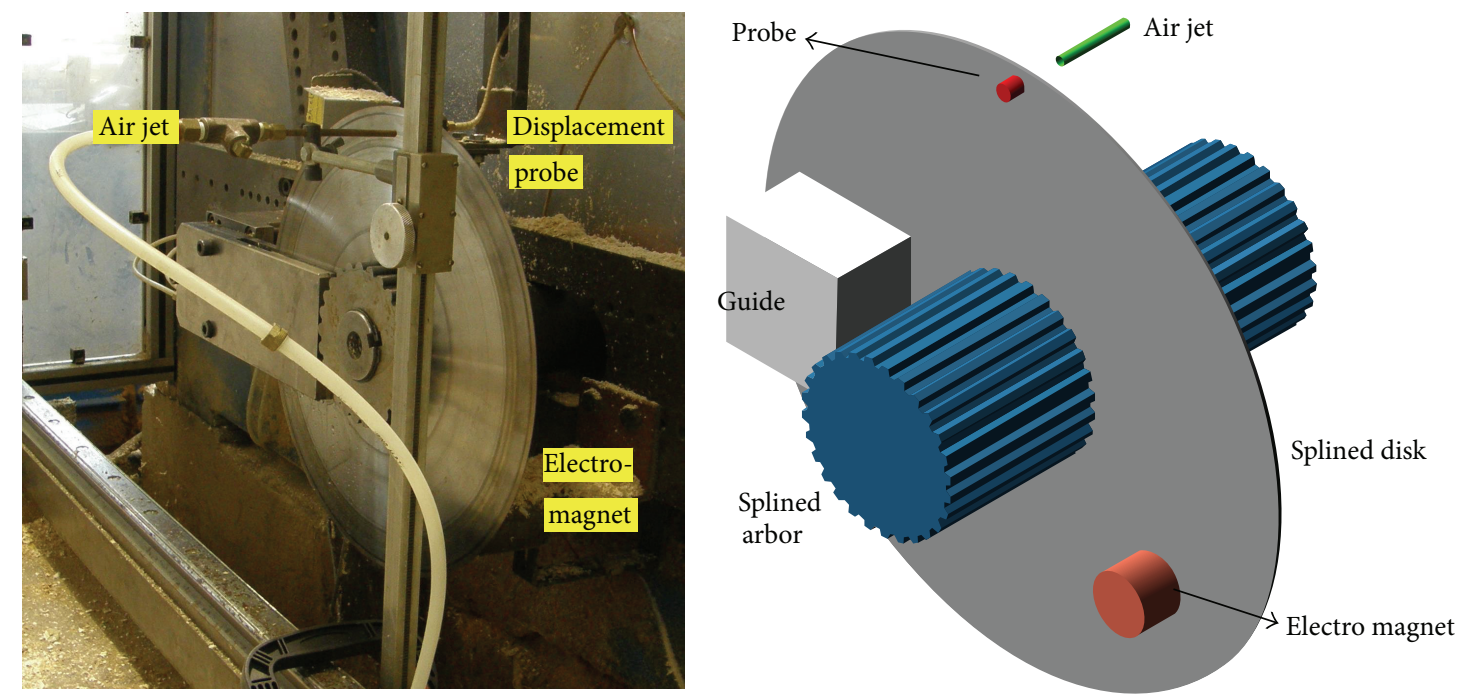

FIGURE 1: Experimental idling tests setup.

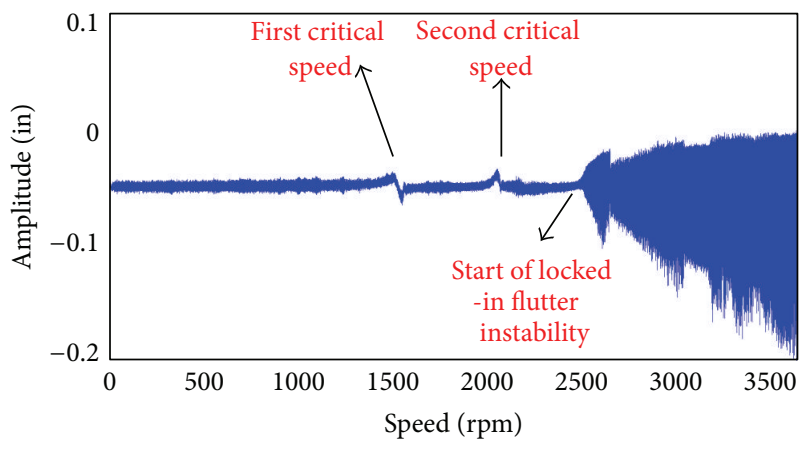

FIgURE 2: Deflection of the disk, during idling run-up from 0 to $3600 \mathrm{rpm}$, measured by displacement probe.

From the time domain and color map two general characteristics of the system can be observed. As the color map indicates, the frequencies (as measured by a space fixed observer) of each mode decrease as the rotation speed increases. At minor critical speeds the frequency of a mode reaches zero. Flutter happens at supercritical speeds where a reflected wave frequency coincides with a backward travelling wave frequency. For example, Figure 3 illustrates several points where reflected waves of mode 2 and 3 meet the backward waves of modes 2,3 , and 4 . The results indicate that by increasing the rotation speed system could pass these flutter zones. But at speed about $2500 \mathrm{rpm}$, when the reflected wave of mode 1 reaches the backward wave of mode 4 the system cannot pass the flutter speed. We define the term "locked-in flutter instability" where the system cannot pass a flutter zone. After this speed there are several lockin frequency paths in which the frequencies are constant and independent of rotation speed. The maximum stable operation speed of a guided splined disk is defined as the start of a locked-in flutter. One possible reason is that when system reaches to this flutter, the deflection of the system is large so that system cannot pass it.
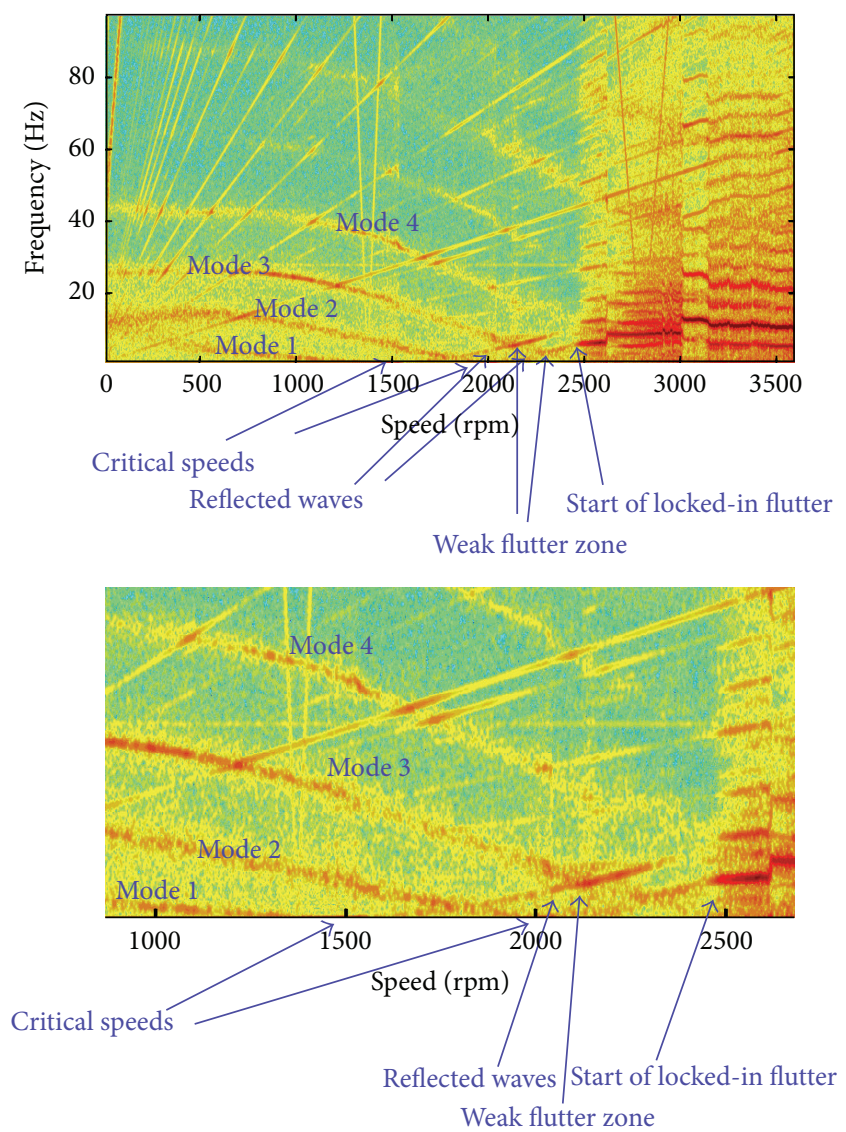

FIgURE 3: Variation of excited frequencies of the disk as a function of rotation speed.

\section{Equations of Motions and Numerical Results}

Figure 4 shows the idealized representation of a splined guided disk. The transverse displacement of the disk is $w$, 


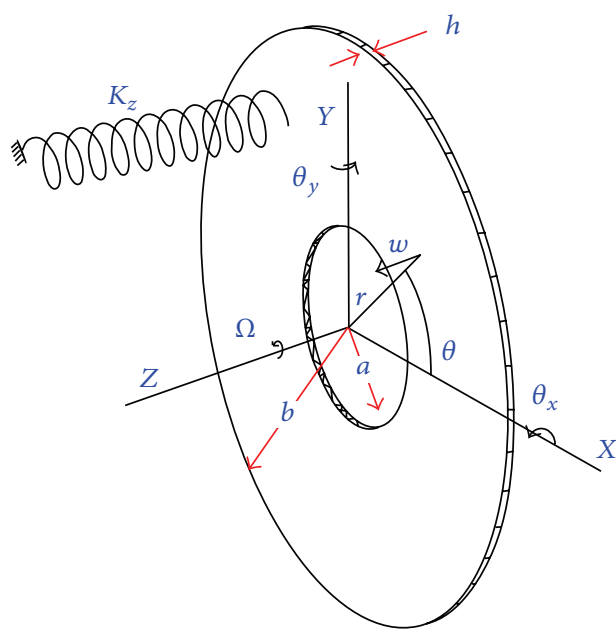

FIGURE 4: Spinning disk with free inner and outer boundary conditions, with rigid body tilting and translational motions, constrained by a lateral spring.

measured with respect to stationary coordinates $(r, \theta)$. The rigid body modes of the disk are defined by $Z, \theta_{x}$, and $\theta_{y}$. The guides are represented by a linear spring of stiffness $k_{z}$ located at $\left(r_{k}, \theta_{k}\right)$.

The governing linear equation of motion is [8]

$$
\begin{aligned}
\rho h\left(w_{, t t}+2 \Omega w_{, t \theta}+\Omega^{2} w_{, \theta \theta}\right)+D \nabla^{4} w-\frac{h}{r}\left(\sigma_{r r} r w_{, r}\right)_{, r} \\
-\frac{h}{r^{2}} \sigma_{\theta \theta} w_{, \theta \theta}+\rho h \ddot{Z}+\rho h\left(r \ddot{\theta}_{x} \sin \theta-r \ddot{\theta}_{y} \cos \theta\right) \\
+\rho h\left(2 \Omega r \cos \theta \dot{\theta}_{x}+2 \Omega r \sin \theta \dot{\theta}_{y}\right) \\
=-\frac{k_{z}}{r}\left(w+Z+r_{k} \sin \theta_{k} \theta_{x}-r_{k} \cos \theta_{k} \theta_{y}\right),
\end{aligned}
$$

where $h, \rho, \Omega, D, E$, and $v$ are thickness, mass density, constant rotating speed, flexural rigidity, Young's modulus, and Poisson's ratio. $\sigma_{r r}$ and $\sigma_{\theta \theta}$ are symmetric stresses. The closed form solution of $\sigma_{r r}$ and $\sigma_{\theta \theta}$, for a disk with free inner boundary condition, can be found in [1].

The equations governing rigid body tilting are [8]

$$
\begin{aligned}
& \frac{\pi}{4} \rho h\left(b^{4}-a^{4}\right)\left(\ddot{\theta}_{x}+2 \Omega \dot{\theta}_{y}\right) \\
& \quad+\int_{0}^{2 \pi} \int_{a}^{b} \rho h \sin \theta\left(w_{, t t}+2 \Omega w_{, t \theta}\right) r^{2} d r d \theta \\
& =k_{z}\left(w+Z+r_{k} \sin \theta_{k} \theta_{x}-r_{k} \cos \theta_{k} \theta\right) r_{k} \sin \theta_{k} \\
& \frac{\pi}{4} \rho h\left(b^{4}-a^{4}\right)\left(\ddot{\theta}_{y}-2 \Omega \dot{\theta}_{x}\right) \\
& \quad+\int_{0}^{2 \pi} \int_{a}^{b} \rho h \cos \theta\left(w_{, t t}+2 \Omega w_{, t \theta}\right) r^{2} d r d \theta \\
& =-k_{z}\left(w+Z+r_{k} \sin \theta_{k} \theta_{x}-r_{k} \cos \theta_{k} \theta_{y}\right) r_{k} \cos \theta_{k} .
\end{aligned}
$$

TABLE 1: The physical properties of the disk under investigation.

\begin{tabular}{lc}
\hline Physical property & Value \\
\hline Outer diameter (Rim) & $700 \mathrm{~mm}(28 \mathrm{in})$ \\
Inner diameter (Eye) & $200 \mathrm{~mm}(8 \mathrm{in})$ \\
Thickness & $2.8 \mathrm{~mm}(0.110 \mathrm{in})$ \\
Density & $7800\left(\mathrm{Kg} / \mathrm{m}^{3}\right)$ \\
Young's modulus & $203(\mathrm{GPa})$ \\
Poisson's ratio & 0.3 \\
\hline
\end{tabular}

The rigid body translational equation in $Z$ direction is

$$
\begin{aligned}
& \rho h\left(b^{2}-a^{2}\right) \ddot{Z}+\int_{0}^{2 \pi} \int_{a}^{b} \rho h\left(w_{, t t}\right) r d r d \theta \\
& =-k_{z}\left(w+Z+r_{k} \sin \theta_{k} \theta_{x}-r_{k} \cos \theta_{k} \theta_{y}\right) .
\end{aligned}
$$

Solution of the equations was obtained by application of the Galerkin method. In this solution method the eigenfunction of a stationary disk was used as the approximation function for the Galerkin method. The solution method for solving the equations and mathematical procedure can be found in Appendix B.

In this section, the imaginary and real parts of the eigenvalues are computed numerically for a disk with physical properties summarized in Table 1 . The disk is constrained by 16 lateral springs each of stiffness $k=10^{5} \mathrm{~N} / \mathrm{m}$ distributed over a $200 \times 200 \mathrm{~mm}$ square area which represents the guides.

Figure 5 shows the eigenvalues of the disk as a function of rotation speed. Comparing the natural frequency graph predicted by the numerical analysis and those obtained from experimental results (the color map Figure 3) indicates that there is a good agreement between the numerical results and experimental results. From the numerical results the start of flutter instability of interaction between mode 4 and reflected wave of mode 1 is $2510 \mathrm{rpm}$, which is about the start of lockedin flutter speed obtained from the experimental results during run-up tests.

The Eigenvalues of a spinning disk can be pure imaginary, a real value, or a complex number. Figure 5 shows the imaginary and real parts of eigenvalues for the guided splined spinning disk under investigation.

The imaginary part of eigenvalues controls the frequency of transverse vibrations, and the real part controls the amplitude of transverse vibrations of the disk. At critical speed, when the natural frequency of a mode tends to zero, the eigenvalue bifurcates from pure imaginary to pure real. The type of instability here is known as "divergence."

Flutter, also termed lock-in, is the merging of two nonzero natural frequencies to form a complex conjugate pair. Such behavior occurs when two modes interact, typically due to coupling. Two modes can be coupled by a mean to flow kinetic energy between them. For a spinning disk it can occur by adding a mass or a lateral constraint such as a spring. In this case, application of lateral springs (representation of the guides) provides the coupling between modes. Merging of two modes occurs when one natural frequency decreases and one increases. Figure 5 indicates that, at supercritical speeds, 


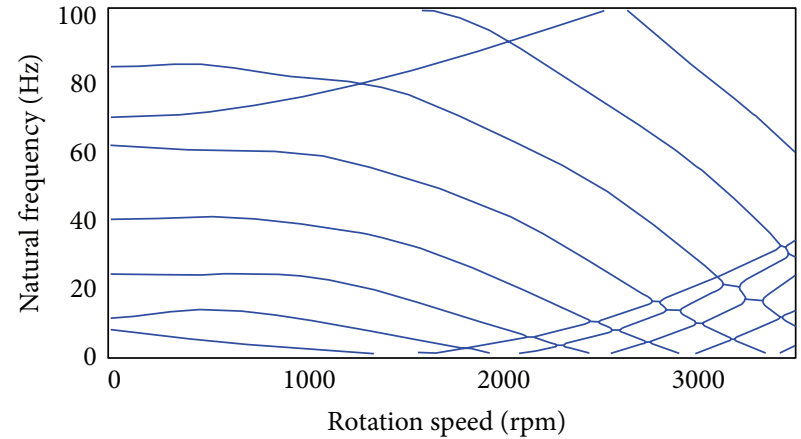

(a)

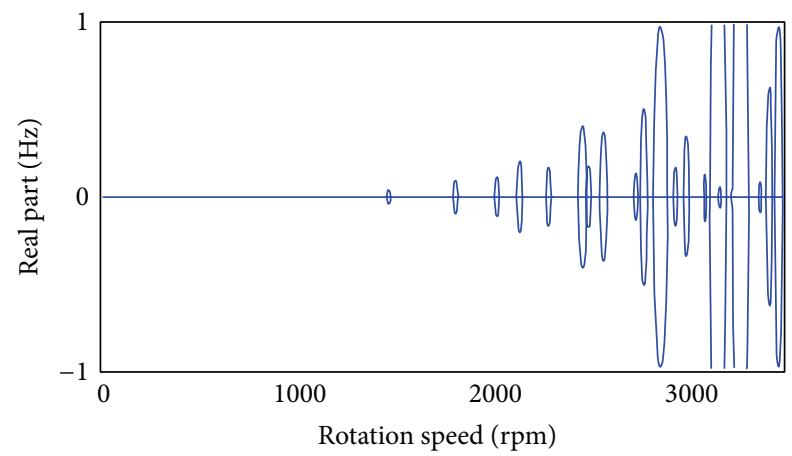

(c)

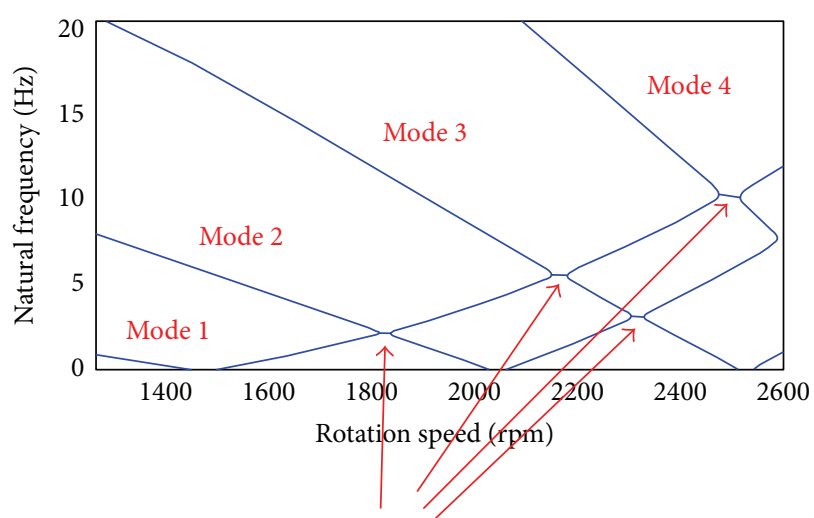

Flutter instability zones

(b)

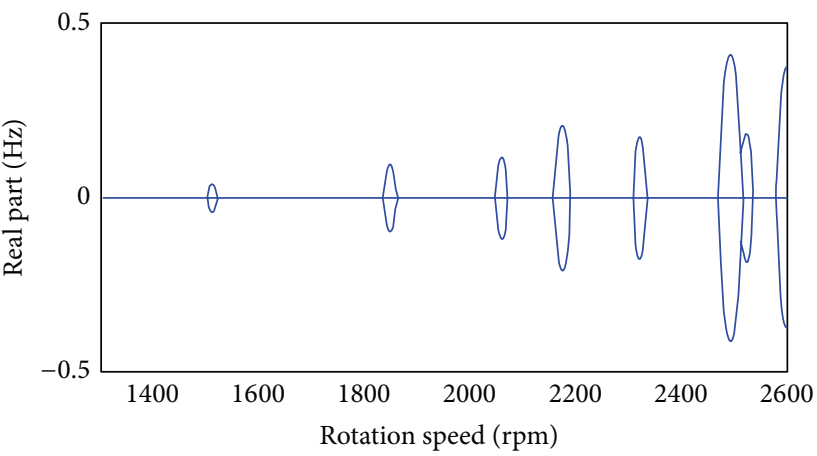

(d)

FIGURE 5: Imaginary and real parts of eigenvalues of the guided splined disk.

when a reflected wave meets a backward wave of different mode, the two frequency lines merge together. Figure 5 also indicates that when the two modes merge together the real part of eigenvalues is positive. In other words, the system is unstable, which is referred to as flutter instability. At the onset of flutter two distinct imaginary solutions merge into a complex conjugate pair and then they unlock. The slope of frequency line in region between the two bifurcations can be positive, negative, or zero, depending on the inertial and elastic forces of the two modes merging together. If the coupling is strong, the coupling forces are larger than elastic and inertial forces in the individual mode, the system experiences lock-in frequency, and natural frequency is independent of rotation speed. In fact, here because the coupling forces (the lateral constrains by the guides) are greater than the inertia and elastic forces of each mode, then the coupling forces are dominant. Since the coupling forces are independent of rotation speed, then the system experiences lock-in frequency, in the region where flutter instability occurs.

The color map (Figure 3) illustrates other excited frequencies in the system which analysis did not show. This is due to the fact that there are imperfections in the balance and interaction of the components of practical rotating machines. As a result of these imperfections, rotating parts of machines generate vibration. Common sources of such vibrations arise due to mechanical looseness, mass unbalance, eccentricity, misalignments, bent shaft, pulleys, external forces, and rubbing. These faults usually yield vibration levels with harmonics of $1 X, 2 X, 3 X, \ldots$ and subharmonics levels of vibration such as $X / 2, X / 3, \ldots$ of the rotation speed which can be seen in the color map. If the frequency of excitation generated by a rotating part coincides with the natural frequencies of the rotating blade, resonant interaction can be expected.

\section{The Effect of Geometrical Properties of the System on the Start of Locked-In Flutter Instability Speeds}

A nondimensionalized analysis of the speed of a spinning disk indicates that the start of flutter has a linear relationship with disk thickness (for details see Appendix A):

$$
\Omega=\sqrt{\frac{D}{\rho h b^{4}}} \Omega^{*},
$$

whereas $D$ is the flexural rigidity

$$
D=\frac{E h^{3}}{12\left(1-v^{2}\right)} \text {. }
$$




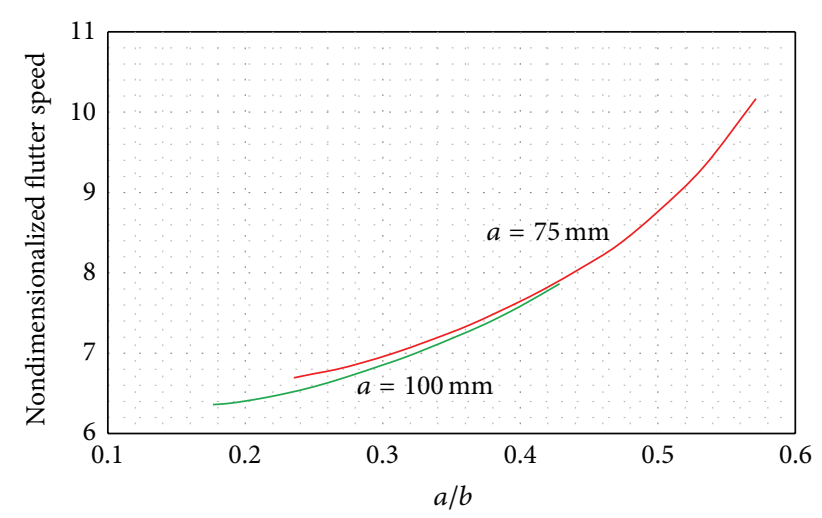

Figure 6: Nondimensionalized locked-in flutter speed.

Therefore,

$$
\Omega=\sqrt{\frac{E}{12 \rho\left(1-v^{2}\right)}} \frac{h}{b^{2}} \Omega^{*},
$$

where $\Omega^{*}$ is the nondimensionalized rotation speed and is a function of the value of $a / b$.

Figure 6 shows the nondimensionalized lock-in flutter speed as a function of $a / b$ for $a=75 \mathrm{~mm}(3 \mathrm{in})$ and $a=$ $100 \mathrm{~mm}$ (4 in).

\section{Conclusion}

Experimental run-up tests of a guided splined disk indicate that flutter instability happens at speeds when a backward wave of a mode meets a reflected wave of another mode at supercritical speeds. At lower rotation speed the system can pass a flutter zone, but there is a speed above which the system cannot pass flutter speed and transverse vibrations of the disk lock into that flutter instability zone.

The governing linear equations of transverse motion of a guided splined spinning disk with a free inner boundary condition and constrained from lateral motion by linear springs can be used to predict the flutter instability zones. However, this mathematical model fails to predict the behaviour of the system after it locks into a flutter speed. A possible explanation is the geometrical nonlinearity of the system at postflutter speeds due to large transverse deflection of the disk. Therefore, for further analyses of the guided splined disk study of nonlinear equations of motion should be undertaken.

\section{Appendices}

\section{A. Mathematical Calculation of Nondimensional Equation of Motion}

Nondimensional parameters are introduced as

$$
R=\frac{r}{b}, \quad W=\frac{w}{b}, \quad \tau=T t,
$$

where $T=\sqrt{D / \rho h b^{4}}$.
Based on the introduced parameters,

$$
\begin{gathered}
\Omega^{*}=\frac{\Omega}{T}, \\
\frac{\partial w}{\partial t}=\frac{\partial(b W)}{\partial(\tau / T)}=T b \frac{\partial W}{\partial \tau}, \quad \frac{\partial^{2} w}{\partial t^{2}}=T^{2} b \frac{\partial^{2} W}{\partial \tau^{2}} \\
\frac{\partial^{2} w}{\partial t \partial \theta}=T b \frac{\partial^{2} W}{\partial \tau \partial \theta}, \quad \nabla^{4} w=\frac{1}{b^{3}} \nabla^{4} W \\
\frac{\partial^{2} w}{\partial \theta^{2}}=b \frac{\partial^{2} W}{\partial \theta^{2}}, \quad \frac{\partial w}{\partial r}=\frac{\partial(b W)}{\partial(b R)}=\frac{\partial W}{\partial R}, \\
\frac{\partial^{2} w}{\partial r^{2}}=\frac{\partial}{\partial(b R)} \frac{\partial w}{\partial r}=\frac{1}{b} \frac{\partial^{2} W}{\partial R^{2}} .
\end{gathered}
$$

By substitution of, $\sigma_{r}$, and $\sigma_{\theta}$ into equation of motion, we get

$$
\begin{gathered}
\rho h\left(w_{, t t}+2 \Omega w_{, t \theta}+\Omega^{2} w_{, \theta \theta}\right)+D \nabla^{4} w \\
-\frac{h}{r} \rho \Omega^{2}\left(w_{, r}\left(C_{1} r+\frac{C_{2}}{r}+C_{3} r^{3}\right)\right)_{, r} \\
-h \rho \Omega^{2}\left(\frac{C_{1}}{r^{2}}-\frac{C_{2}}{r^{4}}+C_{4}\right) w_{, \theta \theta}=0 .
\end{gathered}
$$

By simplification of (A.3) the equation may thus be written in the form of:

$$
\begin{array}{r}
\rho h\left(w_{, t t}+2 \Omega w_{, t \theta}+\Omega^{2} w_{, \theta \theta}\right)+D \nabla^{4} w \\
-h \rho \Omega^{2}\left(w_{, r r}\left(C_{1}+\frac{C_{2}}{r^{2}}+C_{3} r^{2}\right)\right. \\
\left.+w_{, r}\left(\frac{C_{1}}{r}-\frac{C_{2}}{r^{3}}+3 C_{3} r\right)\right) \\
-h \rho \Omega^{2}\left(\frac{C_{1}}{r^{2}}-\frac{C_{2}}{r^{4}}+C_{4}\right) w_{, \theta \theta}=0 .
\end{array}
$$

Here by substituting the nondimensional parameters into (A.4),

$$
\begin{array}{r}
\rho h T^{2} b\left(W_{, \tau \tau}+2 \Omega^{*^{2}} W_{, \tau \theta}+\Omega^{*^{2}} W_{, \theta \theta}\right)+\frac{D}{b^{3}} \nabla^{4} W \\
-\rho h T^{2} \Omega^{*^{2}}\left[\frac{W_{, R R}}{b}\left(C_{1}+\frac{C_{2}}{R^{2} b^{2}}+C_{3} R^{2} b^{2}\right)\right. \\
\left.+W_{, R}\left(\frac{C_{1}}{R b}-\frac{C_{2}}{R^{3} b^{3}}+3 C_{3} R b\right)\right] \\
-\rho h T^{2} \Omega^{*^{2}} b\left(\frac{C_{1}}{R^{2} b^{2}}-\frac{C_{2}}{R^{4} b^{4}}+C_{4}\right) W_{, \theta \theta}=0 .
\end{array}
$$


By dividing both sides of (A.5), the equation may be written as

$$
\begin{gathered}
\left(W_{, \tau \tau}+2 \Omega^{*^{2}} W_{, \tau \theta}+\Omega^{*^{2}} W_{, \theta \theta}\right)+\frac{D}{\rho h T^{2} b^{4}} \nabla^{4} W \\
-\frac{\rho \Omega^{*^{2}}}{\rho b}\left[\frac{W_{, R R}}{b}\left(C_{1}+\frac{C_{2}}{R^{2} b^{2}}+C_{3} R^{2} b^{2}\right)\right. \\
\left.+W_{, R}\left(\frac{C_{1}}{R b}-\frac{C_{2}}{R^{3} b^{3}}+3 C_{3} R b\right)\right] \\
-\frac{\rho \Omega^{* 2}}{\rho}\left(\frac{C_{1}}{R^{2} b^{2}}-\frac{C_{2}}{R^{4} b^{4}}+C_{4}\right) W_{, \theta \theta}=0 .
\end{gathered}
$$

Defining $D / \rho h T^{2} b^{4}=1$ results in

$$
T=\sqrt{\frac{D}{\rho h b^{4}}} .
$$

By substituting $T$ into (A.6) the equation can be written as

$$
\begin{gathered}
W_{, \tau \tau}+2 \Omega^{*} W_{\tau \theta}+\Omega^{* 2} W_{, \theta \theta}+\nabla^{4} W \\
-\frac{\Omega^{* 2}}{b^{2}}\left(C_{1}+\frac{C_{2}}{R^{2} b^{2}}+C_{3} R^{2} b^{2}\right) W_{, R R} \\
-\frac{\Omega^{* 2}}{b}\left(\frac{C_{1}}{R b}-\frac{C_{2}}{R^{3} b^{3}}+3 C_{3} R b\right) W_{, R} \\
-\frac{\Omega^{* 2}}{1}\left(\frac{C_{1}}{R^{2} b^{2}}-\frac{C_{2}}{R^{4} b^{4}}+C_{4}\right) W_{, \theta \theta}=0 .
\end{gathered}
$$

This equation may be written in the form of

$$
\begin{aligned}
W_{, \tau \tau} & +2 \Omega^{*} W_{, \tau \theta}+\Omega^{* 2} W_{, \theta \theta}+\nabla^{4} W \\
- & \frac{\Omega^{* 2}}{b^{2}}\left(C_{r r} W_{, R R}+C_{r} W_{, R}+C_{\theta \theta} W_{, \theta \theta}\right)=0,
\end{aligned}
$$

where

$$
\begin{aligned}
& C_{r r}=C_{1}+\frac{C_{2}}{R^{2} b^{2}}+C_{3} R^{2} b^{2}, \\
& C_{r}=b\left(\frac{C_{1}}{R b}-\frac{C_{2}}{R^{3} b^{3}}+3 C_{3} R b\right), \\
& C_{\theta \theta}=b^{2}\left(\frac{C_{1}}{R^{2} b^{2}}-\frac{C_{2}}{R^{4} b^{4}}+C_{4}\right) .
\end{aligned}
$$

\section{B. Mathematical Details of Solution for Linear Equations of Motion}

Solution of (1) can be obtained by application of the Galerkin method. In this solution method the eigenfunctions of a stationary disk are used as the approximation function for the Galerkin method. First the eigenfunctions of a stationary disk are calculated. The nondimensionalized stationary equation of motion for a plate in polar coordinate system is

$$
\nabla^{4} W=\lambda^{4} W
$$

Using the boundary conditions the eigenvalues $\lambda_{m n}$ associated with the eigenfunctions can be obtained as [13]

$$
\Phi_{m n}=\Theta(m \theta) R_{m}\left(\lambda_{m n} R\right),
$$

where

$$
\begin{gathered}
\Theta(m \theta)=\left\{\begin{array}{l}
\sin m \theta \\
\cos m \theta
\end{array}\right\}, \\
R_{m}\left(\lambda_{m n} R\right)=\left(e_{1 m} J_{m}\left(\lambda_{m n} R\right)+e_{2 m} Y_{m}\left(\lambda_{m n} R\right)\right. \\
\left.+e_{3 m} I_{m}\left(\lambda_{m n} R\right)+e_{4 m} K_{m}\left(\lambda_{m n} R\right)\right),
\end{gathered}
$$

where $J_{m}, Y_{m}, I_{m}$, and $K_{m}$ are $m$ th order Bessel functions and modified Bessel functions. $e_{1 m}, e_{2 m}, e_{3 m}$, and $e_{4 m}$ are constants which can be obtained by the normalization condition.

For a circular disk the vibration modes can be described by the number of nodal circles $(n)$ and the number of nodal diameters $(m)$. So, the transverse displacement of the disk may be written by a modal expansion as

$$
\begin{aligned}
W & (R, \theta, t) \\
& =\sum_{m, n=0}^{\infty}\left[S_{m n}(\tau) \sin m \theta+C_{m n}(\tau) \cos m \theta\right] R_{m}\left(\lambda_{m n} R\right)
\end{aligned}
$$

Substituting of $W(R, \theta, t)$ from (B.4) into (1) results in

$$
\begin{gathered}
\ddot{S}_{m n}-2 \Omega^{*} m \dot{C}_{m n}+\left(\lambda_{m n}^{4}-m^{2} \Omega^{* 2}\right) S_{m n} \\
-\frac{\Omega^{* 2}}{b^{2}} \pi \sum_{q=0}^{\infty} S_{m q} \Pi_{m q}=0 \\
\ddot{C}_{m n}-2 \Omega^{*} m \dot{S}_{m n}+\left(\lambda_{m n}^{4}-m^{2} \Omega^{* 2}\right) C_{m n} \\
-\frac{\Omega^{* 2}}{b^{2}} \pi \sum_{q=0}^{\infty} C_{m q} \Pi_{m q}=0
\end{gathered}
$$

where

$$
\begin{aligned}
\Pi_{m q}=\int_{a / b}^{1}\left(C_{r r} \frac{d^{2} R_{m}\left(\lambda_{m q} R\right)}{d R^{2}}+C_{r} \frac{d R_{m}\left(\lambda_{m q} R\right)}{d R}\right. \\
\left.\quad-m^{2} C_{\theta \theta} R_{m}\left(\lambda_{m q} R\right) R_{m}\left(\lambda_{m n} R\right)\right) R d R=0 .
\end{aligned}
$$

Equations (B.5) constitute a system of simultaneous differential equation in $S_{m n}(\tau)$ and $C_{m n}(\tau)$, while $m, n=0,1,2, \ldots$, the solution of which determines the transverse displacement of the disk via (B.4). 


\section{Nomenclature}

$\begin{array}{ll}a: & \text { Inner radius of the disk } \\ b: & \text { Outer radius of the disk } \\ D: & \text { Disk rigidity }\left(E h^{3} / 12\left(1-v^{2}\right)\right) \\ E: & \text { Young's modulus } \\ h: & \text { Disk thickness } \\ k: & \text { Stiffness of the spring } \\ m: & \text { Number of nodal diameters } \\ n: & \text { Number of nodal circles } \\ (r, \theta, z): & \text { Space fixed polar coordinate system } \\ \lambda_{m n}: & \text { Eigenvalues for the transverse displacement } \\ \Phi_{m n}: & \text { of the disk } \\ R_{m n}: & \text { Eigenfunction } \\ S_{m n}, C_{m n}: & \text { Mode shape in the radial direction for the } \\ t: & \text { Time } \\ w: & \text { Transverse displacement } \\ \delta_{i j}: & \text { Kronecker delta } \\ v: & \text { Poisson's ratio } \\ \rho: & \text { Mass density } \\ \sigma_{r r}, \sigma_{\theta \theta}: & \text { Radial and hoop stress due to rotation } \\ \sigma_{r}, \sigma_{\theta}: & \text { Radial and hoop stress due to in-plane edge } \\ \Omega: & \text { loads } \\ & \text { Rotation speed (rad/sec). }\end{array}$

\section{Conflict of Interests}

The authors declare that there is no conflict of interests regarding the publication of this paper.

\section{References}

[1] A. Mohammadpanah, Idling and cutting vibration characteristics of guided circular saws [M.S. thesis on Partial Fulfilment], University of British Columbia, 2012.

[2] D. Mu and S. Chen, "Effect of the guides on the lowest critical rotational frequencies of circular saw," Chinese Journal of Mechanical Engineering, vol. 14, no. 2, pp. 166-170, 2001.

[3] R. M. H. Khorasany and S. G. Hutton, "Vibration characteristics of rotating thin disks-part I: experimental results," Journal of Applied Mechanics, Transactions ASME, vol. 79, no. 4, Article ID 41006, 2012.

[4] R. M. H. Khorasany and S. G. Hutton, "Vibration characteristics of rotating thin disks, part II: analytical predictions," Journal of Applied Mechanics, vol. 79, no. 4, Article ID 041007, 10 pages, 2012.

[5] S. M. Vogel and D. W. Skinner, "Natural frequencies of transversely vibrating uniform annular plates," Journal of Applied Mechanics, vol. 32, no. 4, pp. 926-931, 1965.

[6] J.-S. Chen, C.-Y. Hua, and C.-M. Sun, "On the secondary resonance of a spinning disk under space-fixed excitations," ASME Journal of Vibration and Acoustics, vol. 126, no. 3, pp. 422-429, 2004.

[7] K. B. Price, Analysis of the dynamics of guided rotating free centre plates [Ph.D. dissertation], University of California, Berkeley, Calif, USA, 1987.

[8] J.-S. Chen and B. D. Bogy, "Natural frequencies and stability of a flexible spinning disk-stationary load system with rigid-body tilting," Journal of Applied Mechanics, vol. 60, no. 2, pp. 470-477, 1993.

[9] J. S. Chen and C. C. Wong, "Modal interaction in a spinning disk on a floating central collar and restrained by multiple springs," Journal of the Chinese Society of Mechanical Engineers, vol. 17, no. 3, pp. 251-259, 1996.

[10] R. M. H. Khorasany and S. G. Hutton, "An analytical study on the effect of rigid body translational degree of freedom on the vibration characteristics of elastically constrained rotating disks," International Journal of Mechanical Sciences, vol. 52, no. 9, pp. 1186-1192, 2010.

[11] S.-M. Yang, "Vibration of a spinning annular disk with coupled rigid-body motion," ASME Journal of Vibration and Acoustics, vol. 115, no. 2, pp. 159-164, 1993.

[12] R. M. H. Khorasany, A. M. Panah, and S. G. Hutton, "Vibration characteristics of guided circular saws: experimental and numerical analyses," Transactions of the ASME-Journal of Vibration and Acoustics, vol. 134, no. 6, Article ID 061004, 2012.

[13] L. Meirovitch, Principles and Techniques of Vibrations, Prentice Hall, Upper Saddle River, NJ, USA, 1997. 

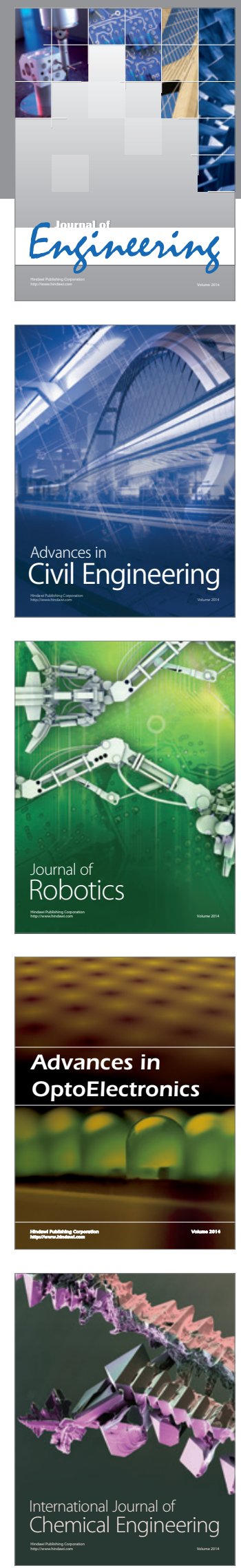

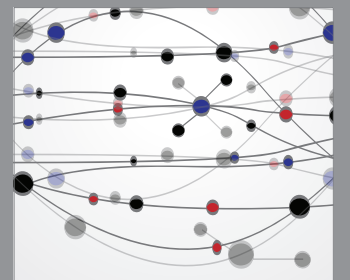

The Scientific World Journal
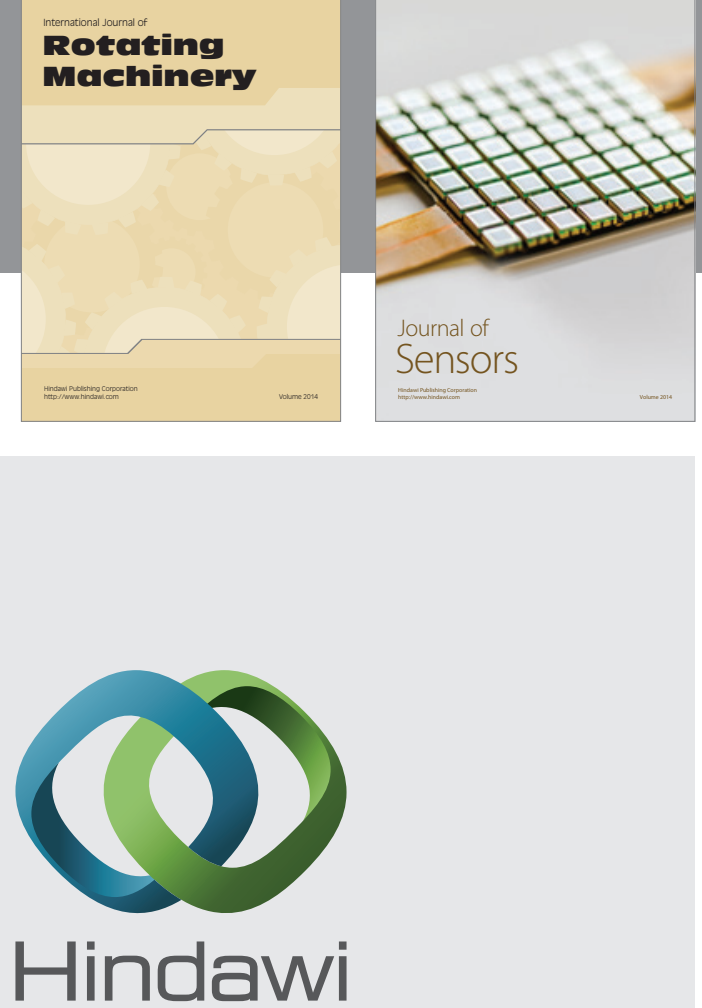

Submit your manuscripts at http://www.hindawi.com
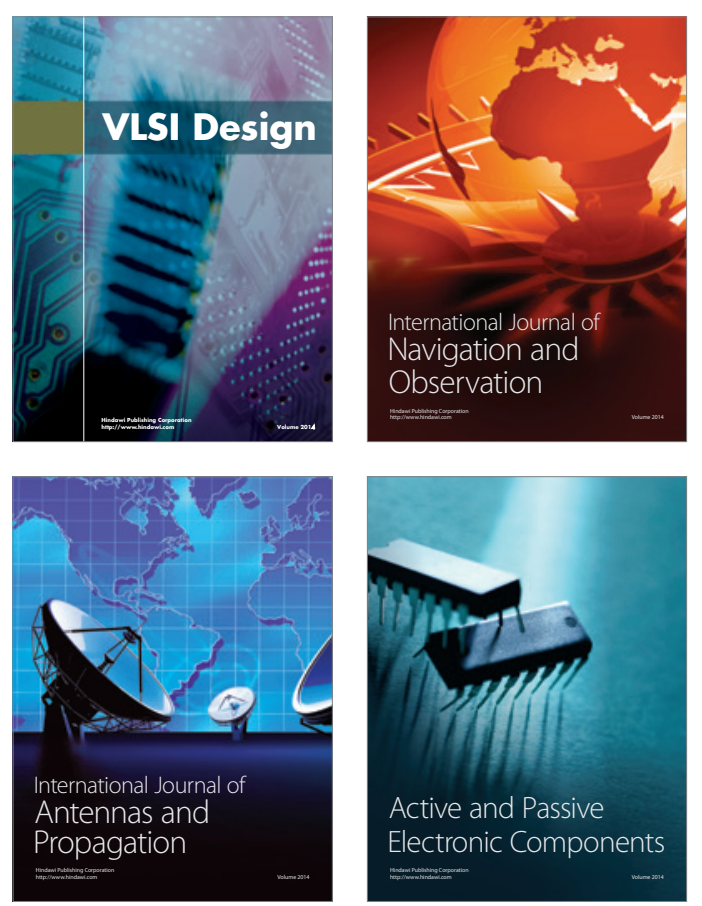
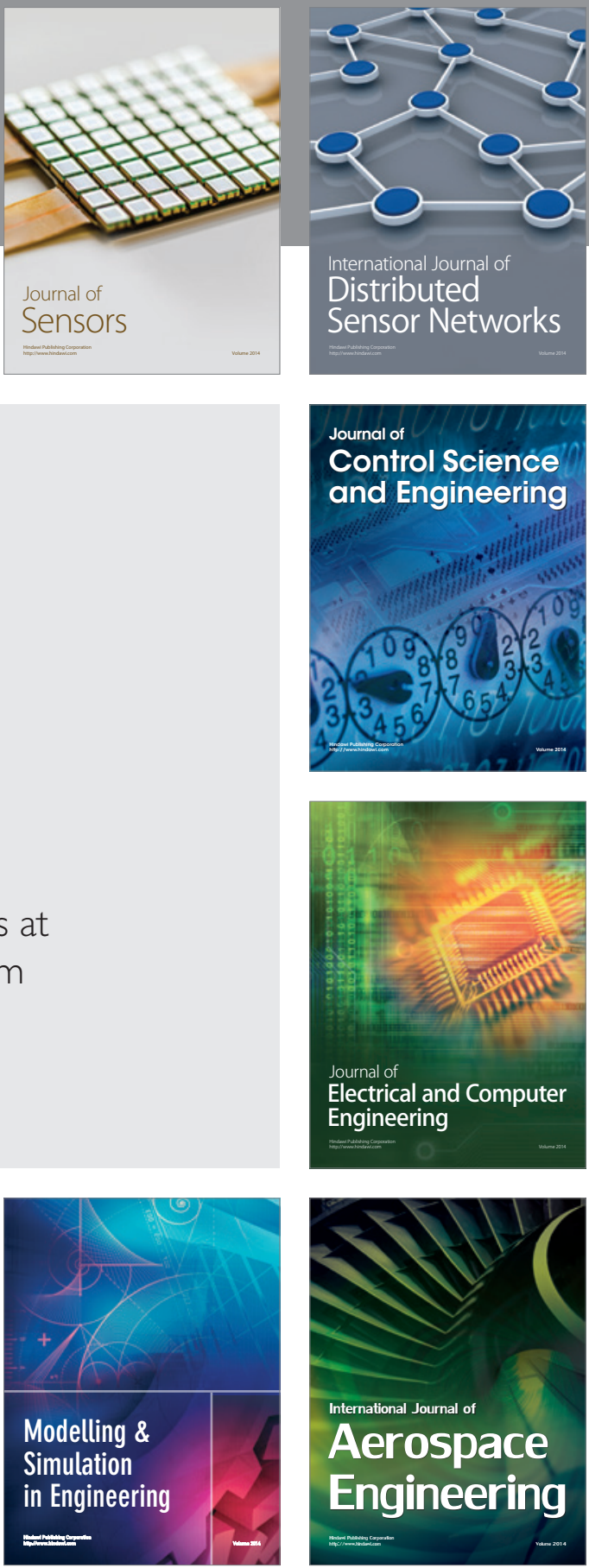

Journal of

Control Science

and Engineering
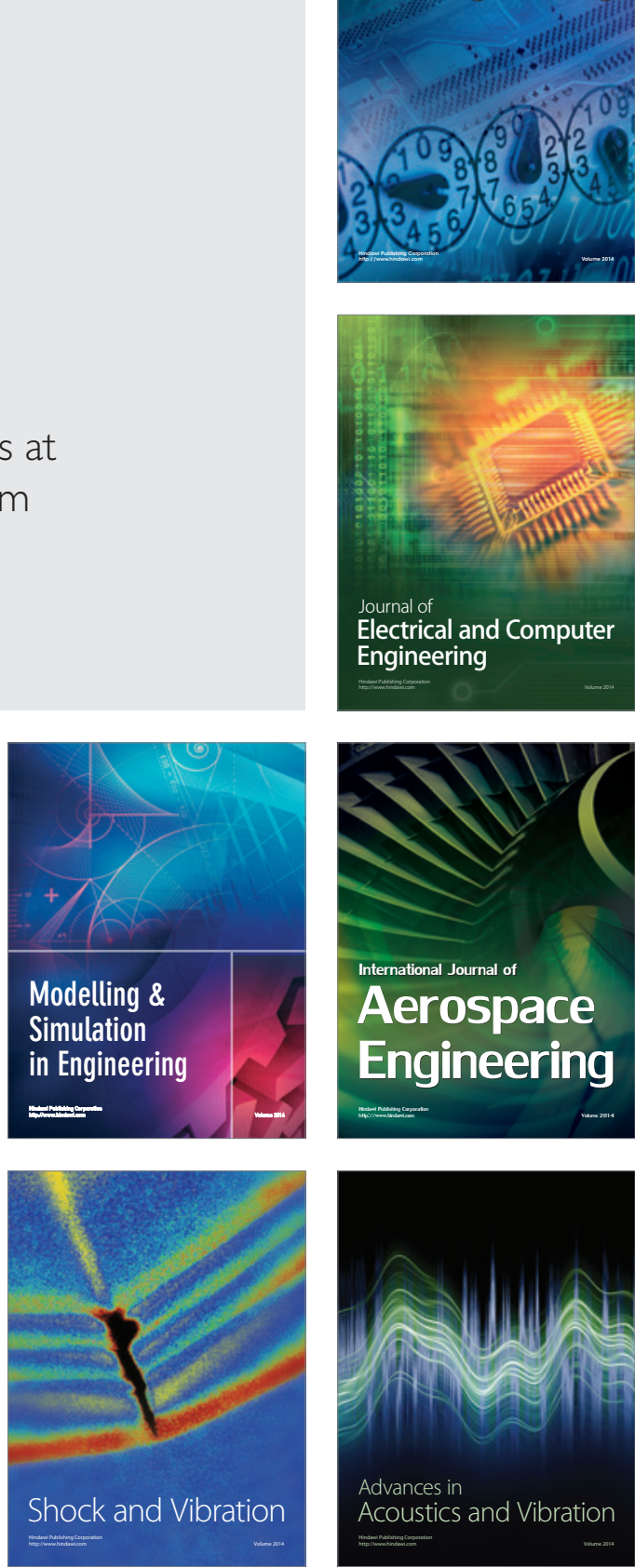